\title{
Movement of Artificial Bacterial Flagella in Heterogeneous Viscous Environments at the Microscale
}

\author{
Kathrin E. Peyer, Famin Qiu, Li Zhang, and Bradley J. Nelson
}

\begin{abstract}
Swimming microrobots have the potential to be used in medical applications such as targeted drug delivery. The challenges for navigating microrobots in the human body lie not only in the viscosity of body fluids but also in the existence of different types of fibers and cells such as blood cells or protein strands. This paper investigates artificial bacterial flagella (ABFs), which are helical microrobots actuated by an external magnetic field, in methyl cellulose solutions of different concentrations. It can be shown that the microrobots can be propelled in these gel-like heterogeneous solutions and successful swimming was demonstrated in solutions with a viscosity of more than 20 times that of water. Furthermore, results indicate that the existence of fibers can help ABFs swim more effectively, which agrees with previous experimental results reported for natural bacteria.
\end{abstract}

\section{INTRODUCTION}

Wirelessly actuated microrobots have great potential to be used in medical applications such as targeted drug delivery or minimally invasive surgery [1]-[3]. There are many different fluid environments in the human body, such as the cerebrospinal fluid, the vitreous humor or blood to name just a few. Microrobots have to be able to navigate in these complex environments (see Fig. 1). Viscosity is a measure of how fluids behave under shear stresses, and Newtonian fluids are characterized by a linear relationship, i.e. constant viscosity, between shear rate and applied shear stress. Fluids that exhibit a non-linear relationship between their shear rate response to shear stresses are called Non-Newtonian fluids.

Body fluids contain molecules and cells that fulfill important functions, e.g. oxygen transport by red blood cells (RBCs). These fibers and microparticles influence the properties of the fluid, such as increasing the viscosity (e.g. blood [4] or cerebrospinal fluid [5]) and influencing the shear response such that the fluid exhibits Non-Newtonian characteristics (e.g. vitreous humor [6]). The challenge is to be able to provide the forces and torques to actuate microrobots in these fluid environments. The microrobots have to overcome the increased viscosity and additional interactions between the microrobotic agent and the fibers can occur (see Fig. 1b inset).

Artificial bacterial flagella (ABFs) are helical microrobots actuated by a uniform rotating magnetic field [7], [8]. They mimic the propulsion mechanism of bacteria such as $E$. coli that use helical flagella for motion generation. Potential applications range from medical application to manipulation

\footnotetext{
*This research was supported by the Swiss National Science Foundation through the National Centre of Competence in Research Robotics.

The authors are with the Institute of Robotics and Intelligent Systems, ETH Zurich, 8092 Zurich, Switzerland \{peyerk, qiuf, lizhang, bnelson\} at ethz.ch.
}

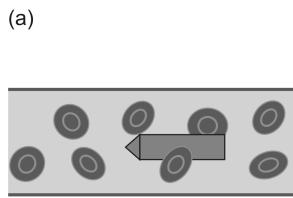

(b)

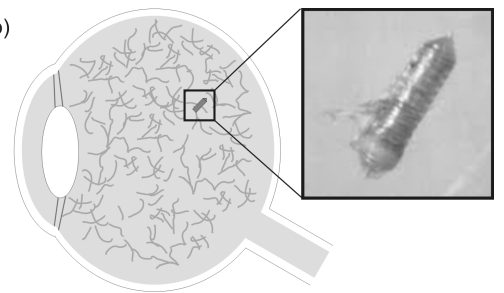

Fig. 1. Challenges for microrobots employed in the human body. (a) Schematic of a robot inside the blood stream. Blood cells can obstruct the propulsion and could potentially become damaged. (b) Schematic of an eye indicating the fibers inside the vitreous humor. Inset shows a $2.4 \mathrm{~mm}$ long screw-type microrobot inside porcine vitreous humor. After several rotations large collagen fibers wrap around the microrobot.

tasks in vitro [9], [10]. Due to their size, both bacteria and ABFs swim at a low Reynolds (Re) number regime, where inertial effects are considered negligible and swimming propulsion is based on the anisotropic drag on the helical filament [11], [12]. In 1979 Berg and Turner showed that $E$. coli and other types of bacteria can swim more efficiently in high viscosity gel-forming fluids than in water [13]. They reasoned that the fibers in the fluid influence the drag anisotropy allowing the filaments to "screw" more efficiently through the liquid than expected from the macroscopically measured viscosity [14], [15]. Recent publication have shown that swimming in viscoelastic, i.e. Non-Newtonian, fluids can also enhance the propulsion efficiency of microorganisms [16], [17].

Methyl cellulose (MC) is a molecule with long unbranched polymers which creates a gel-like fluid when mixed with water. At low concentrations, MC solutions are considered Newtonian, but at concentrations larger than $0.5 \% \mathrm{w} / \mathrm{v}$ the MC solutions become Non-Newtonian [18]. Berg and Turner's results showed that E. coli bacteria swim more efficiently even at concentrations of MC below $0.5 \%$ w/v. This paper investigates $\mathrm{ABFs}$ in three $\mathrm{MC}$ solutions of different concentrations ranging from $0.2 \% \mathrm{w} / \mathrm{v}$ to $0.6 \% \mathrm{w} / \mathrm{v}$. The viscosities, measured at low shear rates, range from 4.9 to $22.1[\mathrm{mPa} \cdot \mathrm{s}]$ and successful swimming could be shown in all three environments. The results are promising when compared to the viscosities expected in some body fluid environments, such as cerebrospinal fluid or blood, which have a viscosity of approximately 1 and $4[\mathrm{mPa} \cdot \mathrm{s}]$, respectively [4], [5]. 


\section{VISCOUS ENVIRONMENTS}

\section{A. Newtonian and Non-Newtonian Fluids}

The viscosity $\eta[\mathrm{Pa} \cdot \mathrm{s}]$ of a liquid represents the relationship between the shear stress $\tau[\mathrm{Pa}]$ and shear rate $\partial u / \partial y$ [1/s] in a fluid (see Fig. 2a):

$$
\tau=\eta \frac{\partial u}{\partial y}
$$

A liquid is considered Newtonian when $\eta$ remains constant and Non-Newtonian if $\eta$ changes. The viscosity can change in different ways, and different types of NonNewtonian fluids can be defined, such as 'shear-thinning' and 'shear-thickening' fluids or 'viscoplastic' and 'viscoelastic' fluids, etc. (see Fig. 2b). The reason for Non-Newtonian behavior can be found in the microscopic structure within the fluids. For example, they may contain polymer chains, large molecules, fibers or microparticles. These structures can become deformed, stretched, or re-aligned and, hence, bias the response of the fluid under shear forces [19]. A simple means to measure viscosity is by applying a shear rate between two parallel plates and measuring the shear forces. In order to characterize viscoelastic fluids, oscillating measurements are used to determine both the plastic and elastic properties of such complex fluids.

\section{B. Fluids in the Human Body}

There are many areas in the human body where microrobots can be employed, e.g. in the spine and brain (cerebrospinal fluid), in the urinary tract, blood stream, or human eye (vitreous humor) [1]. Body fluids often contain molecules and cells which influence the viscosity. The concentration of proteins and red blood cells, for example, has been shown to influence the viscosity of cerebrospinal fluid [5]. Blood can be modeled with a Newtonian viscosity for flows in large arteries, but this approximation fails to hold for the flow in capillaries where the diameter of the blood vessel is similar in scale to the individual RBCs. The vitreous humor is an even more complex fluid, exhibiting viscoelastic properties and containing collagen fibers [2], [6].

\section{Motion in Heterogeneous Fluids}

It is important to consider both the macroscopic viscosity of fluids as well as the interactions at a molecular and microstructural level. Berg and Turner reported that Leptospira, a slender helical bacterium, can swim faster in Non-Newtonian than in Newtonian fluids. Furthermore, they presented experimental data showing that $E$. coli bacteria move with increasing efficiency in MC solutions of increasing concentrations [13]. By considering the microscopic structure of the fluids, this interesting result was attributed to the following effects. First, these bacteria are small enough to fit through the fiber network and swim as if they were in the low viscosity solvent rather than experiencing the macroscopic viscosity of the solution. This alone would not account for an increase in efficiency. Additionally, the fibers provide a means for the bacteria to "push" themselves forward, resulting in a motion
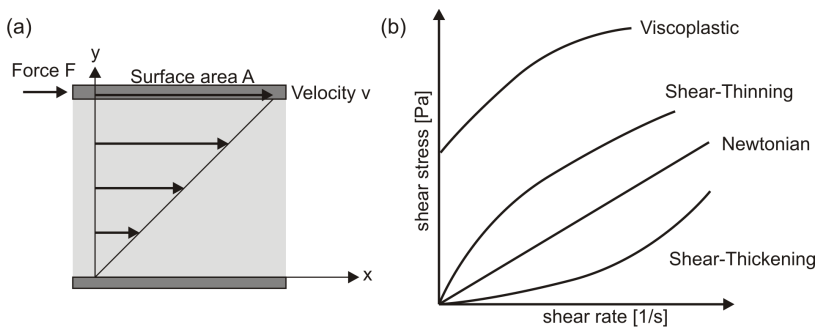

Fig. 2. Viscosity of fluids. (a) A schematic of a simple shear flow between a stationary (bottom) and moving (top) plate. The shear stress $\tau$ is given by the force $F$ applied over an area $A$. (b) Newtonian (linear) and NonNewtonian types of shear stress to shear rate relationships.

that is more similar to the motion of a corkscrew in solid matter rather than in a fluid [13]-[15] (see Fig. 3).

\section{METHODS}

\section{A. Methyl Cellulose Preparation}

A stock solution of MC in deionized (DI) water with a mass concentration of $1 \% \mathrm{w} / \mathrm{v}$ was prepared. First, $300 \mathrm{ml}$ of DI water at $65^{\circ} \mathrm{C}$ was mixed with $3 \mathrm{~g}$ of $\mathrm{MC}$ (Sigma Aldrich). The mixture was stirred vigorously for a few seconds to wet all the MC. Then the mixture was stirred overnight at approximately $300 \mathrm{rpm}$ at room temperature. The desired mass concentrations of $0.2,0.4$ and $0.6 \% \mathrm{w} / \mathrm{v}$ were achieved by diluting the $1 \% \mathrm{w} / \mathrm{v}$ MC solution with DI water. In order to minimize the number of large fibers in the mixture and to enhance the homogeneity of the solution, the MC solutions were filtered. A rough filtering was first performed with a paper coffee filter. Subsequently, a filter with a pore size of 4 $5 \mu \mathrm{m}$ (Schlichter\&Schuell Rundfilter) was used. The mixture was stored at $2^{\circ} \mathrm{C}$.

\section{B. ABF Fabrication}

The ABFs were fabricated using a 3D direct laser writing (DLW) [20] tool by Nanoscribe $\mathrm{GmbH}$ according to the detailed fabrication method presented by Tottori et al. [21]. Fig. 4a shows the typical steps of the ABF fabrication. First, a droplet of IP-L (Nanoscribe $\mathrm{GmbH}$ ) is placed on a glass slide and inserted into the DLW machine. The IP-L solidifies at the focal point of the laser by two-photon-polymerization (TPP). The piezo-actuated stage can be moved in three dimensions to achieve arbitrary designs. In a second step, the sample is rinsed with isopropyl alcohol and dried. In a final step, a 100nm layer of nickel and a 5nm layer of titanium are deposited by electron beam evaporation. Fig. 4b shows a scanning electron micrograph (SEM) image of the final array of ABFs.
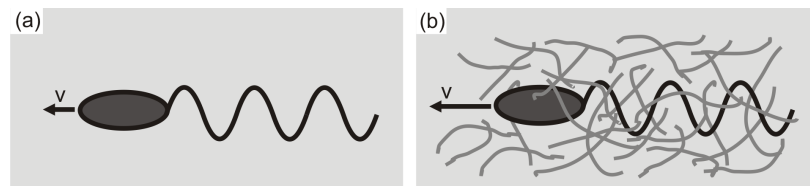

Fig. 3. Schematic representations of E. coli bacteria swimming in (a) water and (b) gel-forming fluids. A bacterium can exploit the fiber structures inside the gel-type fluid and swim more efficiently than in water [13]. 

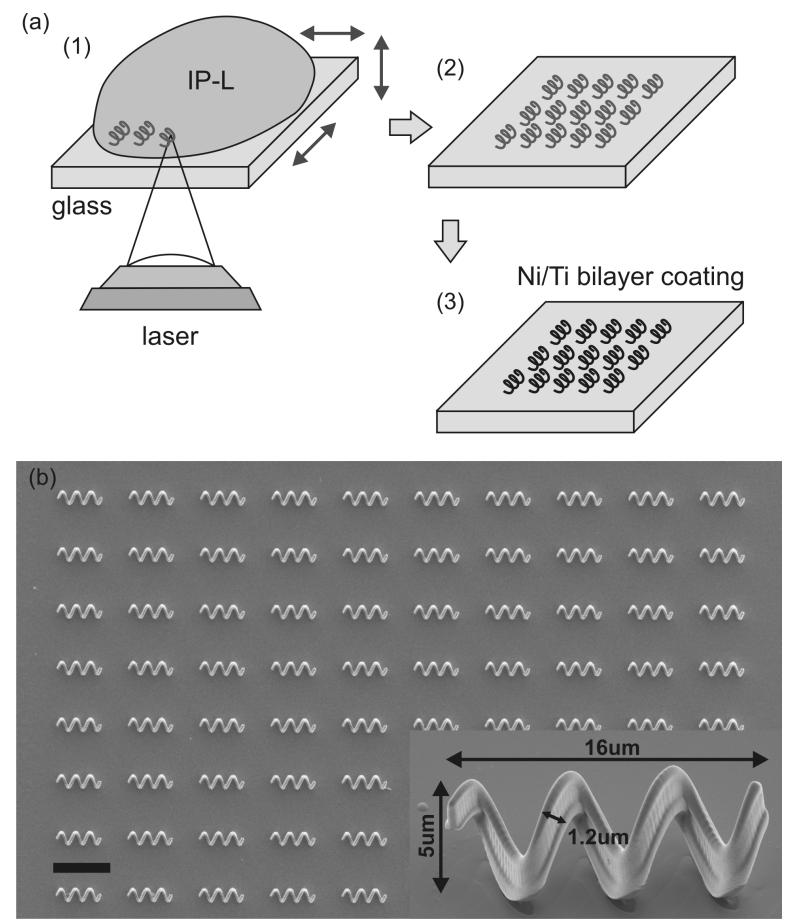

Fig. 4. Polymer artificial bacterial flagella. (a) Fabrication of helical microstructures written by a laser. A two-photon polymerization occurs at the focal point, solidifying the IP-L (Nanoscribe GmbH). Arrays of ABFs are written and, after rinsing, a $\mathrm{Ni} / \mathrm{Ti}$ bilayer is deposited by electron beam evaporation. (b) Scanning electron micrograph (SEM) image of an array of fabricated ABFs. The scale bar corresponds to $20 \mu \mathrm{m}$. Inset shows an individual $\mathrm{ABF}$ and its geometrical dimensions.

\section{Actuation of $A B F s$}

ABFs are actuated by uniform rotating magnetic fields. The magnetic field $\vec{B}$ magnetizes the nickel and a torque $\vec{T}_{M}$ is induced that acts to align the magnetization of the $\mathrm{ABF}$ $\vec{M}$ with the magnetic field

$$
\vec{T}_{M}=V \cdot \vec{M} \times \vec{B}
$$

$V$ is the volume of the magnetic material. The magnetization of the nickel coated helical polymer body is not trivial to model, and Tottori et al. [21] presented some experimental data on how helical bodies magnetize. It has been shown previously that $\mathrm{ABFs}$ wobble at low frequencies and high magnetic fields. When they are actuated at high frequencies, however, their swimming motion stabilizes such that they rotate around their helical axis [21], [22].

\section{Experimental Setup and Analysis}

In order to generate uniform rotating magnetic fields a Helmholtz coil setup, which has three orthogonal coil pairs placed around the experimental tank, was used. The tank had dimensions $3 \mathrm{~cm}$ (length) $\times 1.5 \mathrm{~cm}$ (width) $\times 3 \mathrm{~mm}$ (height), and both the substrate with the fabricated microrobot arrays and a clean Si-wafer surface were placed inside next to each other. Individual ABFs were picked and placed manually by a tungsten probe from the array and placed on the $\mathrm{Si}$ surface. The coils and tank were placed under a microscope with a $480 \times 640$ pixel camera mounted for recording. The individual images from the recording were saved together with pixel size and time stamp information. A custom program written in MATLAB was used to track the initial and end position of the robot in the images and calculate the forward velocity. More details on the experimental setup can be found in previous publications [7], [22].

\section{EXPERIMENTAL RESULTS}

\section{A. Viscosity Measurements}

The viscosity of all three MC solutions was measured at room temperature in a rotational viscometer (Physica UDS 200, Paar Physica). Fig. 5 shows the measured shear stresses at different shear rates for the three MC concentrations. According to [18] the MC was expected to be Newtonian at concentrations below $0.5 \% \mathrm{w} / \mathrm{v}$ and Non-Newtonian at higher concentrations. The measurement of the $0.4 \% \mathrm{w} / \mathrm{v} \mathrm{MC}$ shows a linear behavior as expected, however, the $0.2 \% \mathrm{w} / \mathrm{v}$ $\mathrm{MC}$ is not entirely linear and shows a slight shear thickening behavior. The $0.6 \% \mathrm{w} / \mathrm{v} \mathrm{MC}$ shows a nonlinear behavior as expected. To calculate the viscosity of the three solutions, (1) was used with the values from the measurements at the lowest two shear rates. The resulting viscosity values are shown in Table I.

\section{B. Swim Tests}

To test the swim performance of ABFs in the MC, they were actuated at a constant field strength and rotational frequency, and the velocity measured from the video. Variations in the individual robot design, for example due to the microfabrication process, could lead to different swim performances. Hence, each measurement was done several times with three to four microrobot prototypes taken from the same array to minimize the variance. Fig. 6 shows the measured velocities at different magnetic field input frequencies. In each concentration the experiments were run at two different magnetic field strengths of $4 \mathrm{mT}$ and $8 \mathrm{mT}$.

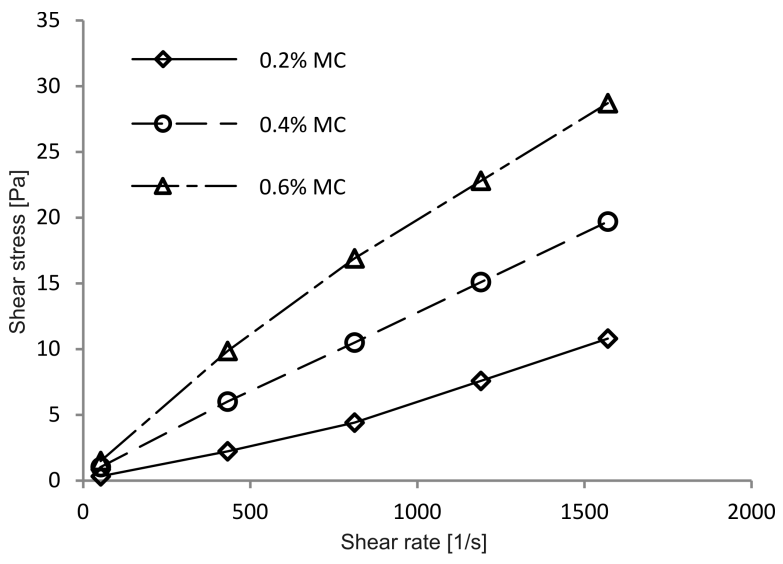

Fig. 5. Viscosity Measurement of Methyl cellulose at different concentrations. \% MC in the legend is short for \% w/v MC in DI water. NonNewtonian behavior was observed at high concentration of $\mathrm{MC}(0.6 \% \mathrm{w} / \mathrm{v})$, but also at low viscosities $(0.2 \% \mathrm{w} / \mathrm{v})$ a shear thickening effect can be discerned. 
TABLE I

VISCOSITY OF MC

\begin{tabular}{c|c} 
MC concentration & Viscosity $[\mathrm{mPa} \cdot \mathrm{s}]$ \\
\hline $0.2 \% \mathrm{w} / \mathrm{v}$ & 4.9 \\
$0.4 \% \mathrm{w} / \mathrm{v}$ & 13.2 \\
$0.6 \% \mathrm{w} / \mathrm{v}$ & 22.1
\end{tabular}

The peak velocity occurs at the stepout frequency, which increases with magnetic field strength and decreases with MC concentration. The slope of the velocity should be constant for different field strengths, which is recognizable in Fig. $6 \mathrm{a}$ and $6 \mathrm{~b}$ for the 0.4 and $0.6 \% \mathrm{w} / \mathrm{v}$ MC concentrations. The experimental results in $0.2 \% \mathrm{w} / \mathrm{v} \mathrm{MC}$ are presented in Fig. 6c. These results exhibit a larger variance in the slope as well as the stepout frequency values.

\section{DISCUSSION}

\section{A. Helical Propulsion Model}

The motion model for helical propulsion at low Reynolds number can be approximated by a 1D 'propulsion matrix' [11]. Instead of looking at the motion in 3D, this model only considers the rotation around and the translation along the helical axis. At low Re numbers inertial effects are considered negligible and a linear relationship between the force and torque and velocity and rotational speed is expected, which is captured in the propulsion matrix:

$$
\left(\begin{array}{l}
F \\
T
\end{array}\right)=\left(\begin{array}{ll}
a & b \\
b & c
\end{array}\right)\left(\begin{array}{c}
u \\
f
\end{array}\right)
$$

where $F$ and $T$ are an external force and torque, and $u$ and $f$ are the velocity and rotational frequency, respectively. The coefficients $a, b$ and $c$ are scalars and can be found by establishing the force and torque equilibrium in the direction of the helical axis. Note that the frequency in the propulsion matrix (3) is commonly given by $\omega=2 \pi f$ in radians per second. Here, the frequency $f[\mathrm{~Hz}]$ is chosen as the magnetic actuation in the experiments is represented in hertz as well. The factor $2 \pi$ can be thought of being incorporated in the parameters $a, b$ and $c$.

Assuming that there is no external force in the direction of the helical axis, which is reasonable when the microrobot swims horizontally, the first line of (3) can be written as

$$
u=-\frac{b}{a} \cdot f
$$

which shows a linear relationship between the frequency $f$ and velocity $v$, which was observed in the experiments in Fig. 6 in the region before the stepout frequency. The stepout frequency is reached when the drag torque, which increases with rotational frequency, exceeds the maximal applicable magnetic torque $T_{M \max } . T_{M \max }$ is given by (2), hence, is determined by the applied field strength and the robot magnetization, which itself is a function of the material and size of the microrobot. The stepout frequency $f_{\max }$ is therefore

$$
f_{\max }=\frac{a}{a c-b^{2}} \cdot T_{M \max }
$$
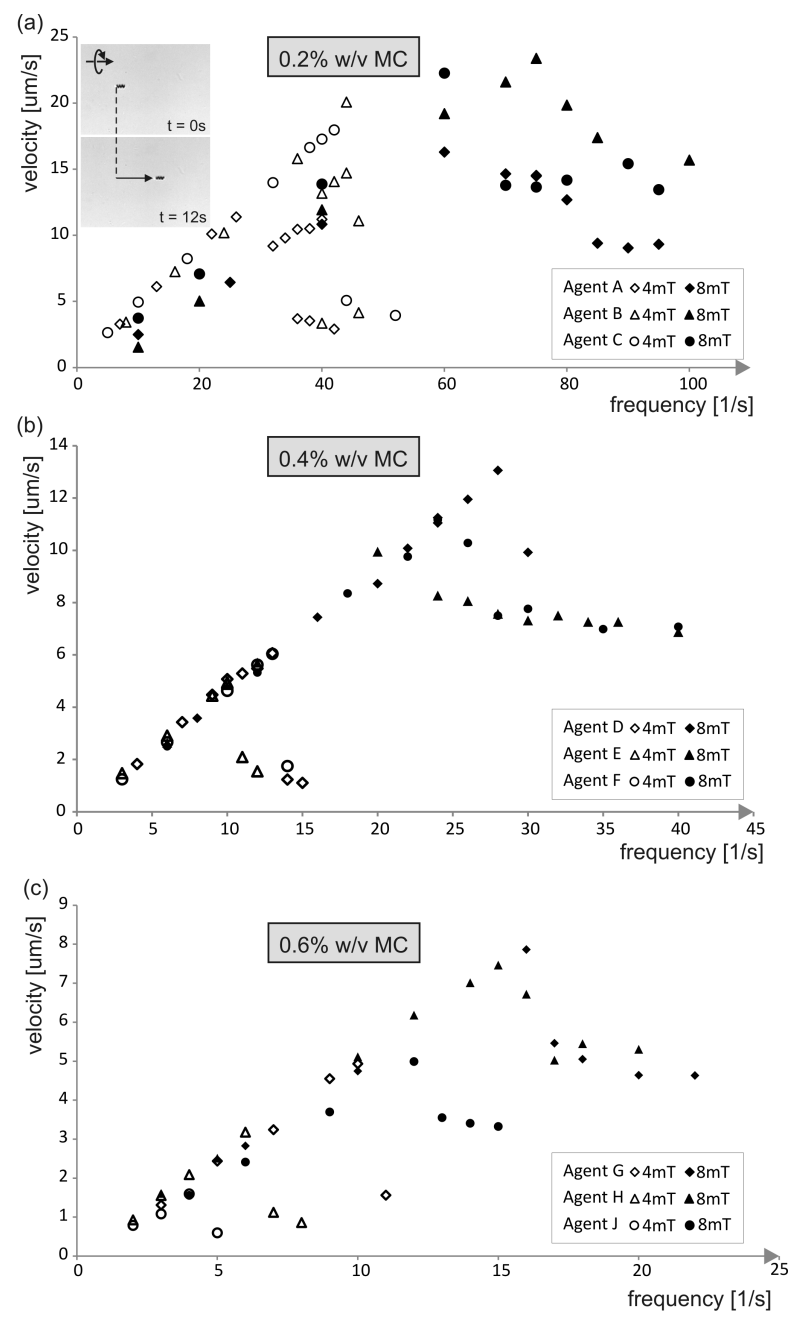

Fig. 6. Swim tests in different $\mathrm{MC}$ concentrations. Swim velocity measurements in (a) $0.6 \% \mathrm{w} / \mathrm{v} \mathrm{MC}$, (b) $0.4 \% \mathrm{w} / \mathrm{v} \mathrm{MC}$ and (c) $0.2 \% \mathrm{w} / \mathrm{v}$. Swim tests were conducted at $4 \mathrm{mT}$ and $8 \mathrm{mT}$ in all three concentrations. At low viscosities higher step-out frequencies can be reached. Inset in (a) shows a video excerpt of a swim experiment, showing the translational motion for a given rotational field input.

The parameters that contribute to $a, b$ and $c$ are the fluid viscosity $\eta$ and the geometrical parameters of the helix, i.e. the helix length and radius, the filament radius, and the helicity angle. For a Newtonian liquid $a, b$ and $c$ are all linearly dependent on the viscosity, such that they can be written as:

$$
\begin{aligned}
& a=\eta \cdot \alpha \\
& b=\eta \cdot \beta \\
& c=\eta \cdot \gamma
\end{aligned}
$$

where $\alpha, \beta$, and $\gamma$ contain all the geometric parameters.

\section{B. Velocity Slope}

It is apparent that for a Newtonian liquid, where (6) is valid, the constant viscosity $\eta$ cancels out in (4) and the slope of the frequency-velocity plot is only a function of the geometric parameters and not of the viscosity

$$
u=-\frac{\beta}{\alpha} \cdot f
$$


For Non-Newtonian fluids or Newtonian fluids containing polymer molecules, however, the assumption of (6) does not necessarily hold true. Fibers in the fluid can change the drag on the helix filament in an inhomogeneous way, which would lead to different apparent viscosities in the coefficients $a, b$, and $c$ such that $b / a \neq \beta / \alpha$ [14].

The slopes of the frequency-velocity plots were measured from the experimental data and the averaged values are shown in Fig. 7. In addition to the data of the swim tests in MC, measurements of the slope of swim tests in DI water are included. The viscosity of water was not measured, but the approximate value of $1 \mathrm{mPa} \cdot \mathrm{s}$ at room temperature was taken for the graph. As mentioned in the experimental section, the velocity slope was not expected to change with magnetic field strengths. In the experiments in water and $0.2 \% \mathrm{w} / \mathrm{v} \mathrm{MC}$ solution, however, a change in the slope is noticeable. This could be attributed to the fact that swimming at high fields and low viscosity enhances the wobbling, a phenomenon reported in previous publications [21], [22], which in turn decreases the forward velocity propulsion.

1) Results in MC: It can be seen that the slopes do not change significantly between the Newtonian $(0.4 \% \mathrm{w} / \mathrm{v})$ and Non-Newtonian MC $(0.6 \% \mathrm{w} / \mathrm{v})$ concentration. Instead, an increase of the slope occurs between the experiments in the lowest viscosity $(0.2 \% \mathrm{w} / \mathrm{v})$ and the experiments in the higher viscosities $(0.4$ and $0.6 \% \mathrm{w} / \mathrm{v})$, which is similar to the results reported for bacteria in viscous environments. This indicates that helical microrobots could not only potentially navigate body fluids, but could possibly do so more efficiently than in fiber-free liquids.

Despite the higher concentration of fibers in the $0.6 \% \mathrm{w} / \mathrm{v}$ solution than the $0.4 \% \mathrm{w} / \mathrm{v}$ solution, the velocity slope remains approximately constant. A possible explanation is that the $0.4 \% \mathrm{w} / \mathrm{v}$ solution is already quite saturated with fibers in comparison to the size of the microrobot. Berg and Turner [13] suggested that E. coli swim with their helical tail through the MC solution as if it were a porous medium. The bacteria are able to move between the fibers of the MC, and in doing so, they do not encounter the macroscopic viscosity of the MC solution, but rather swim or glide in the space between the fibers. Even though the $\mathrm{ABF}$ prototypes presented in this work have a similar overall size compared to $E$. coli bacteria, the filament is thicker by one order of magnitude or more. A single flagellum has a thickness of only $20 \mathrm{~nm}$. While an E. coli bacterium swims, several flagella bundle together which increases the apparent thickness of the helical tail by a small factor. The filament of the $\mathrm{ABF}$, on the other hand, is around $1 \mu \mathrm{m}$, and this filament thickness is difficult to decrease with the TPP fabrication method, as the line resolution is limited. It is therefore perceivable that, due to the larger filament thickness, the ABFs cannot move through the fiber network as easily as bacteria can.

2) Results in water: The velocity slopes in water seem to be higher then in $\mathrm{MC}$, however comparisons have to be made carefully, as the results for water were taken from swim experiments near a solid surface whereas the tests in MC

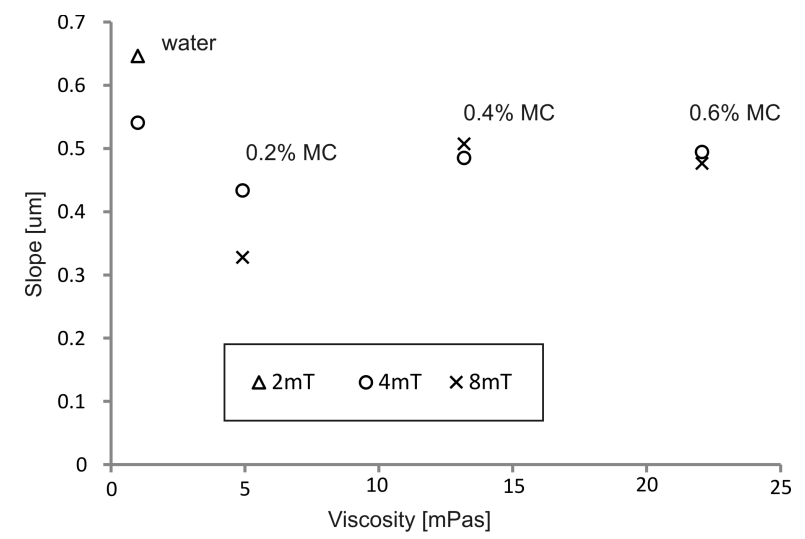

Fig. 7. Slope comparison at different concentrations and field strengths The experimental data in water was taken from swim experiments near a solid surface which increases the slope. The velocity slopes measured in the lowest MC concentration and water are not constant for different field strengths.

were conducted away from the surface. Motion near a solid surface influences the propulsion and the efficiency of helical swimming increases compared to swimming in free space. Furthermore, an apparent drag imbalance near boundaries leads a circular swim trajectory in bacteria [23], or a linear drift in ABFs [22]. In order to compare the swim results in DI to the ones in the MC solutions, more swim tests in water have to be conducted at a larger distance from the substrate surface.

\section{Step-Out Frequency}

The stepout frequency is another important parameter to study. Assuming a Newtonian fluid with constant viscosity, (5) can be rewritten using (6):

$$
f_{\max }=\frac{\alpha}{\eta\left(\alpha \gamma-\beta^{2}\right)} \cdot T_{M \max }
$$

For a given magnetic field and, therefore, maximum magnetic torque, (8) can be written in the simplified form

$$
f_{\text {max }}=\frac{C}{\eta}
$$

where the constant $C$ contains all the parameters $\alpha, \beta, \gamma$ and $T_{\text {Mmax }}$. From (9) it can immediately be seen that the stepout frequency is inversely proportional to the viscosity in a Newtonian fluid. Fig. 8 shows the stepout frequencies for the different MC concentrations and at two different field strengths of $4 \mathrm{mT}$ and $8 \mathrm{mT}$. The average values of the experimental data were taken and the error bars show the standard deviation. The theoretical curve from (9) was plotted with the experimental data for both the experiments at field strengths of $4 \mathrm{mT}$ and the $8 \mathrm{mT}$. The constant $C$ was calculated using the data point at $0.4 \% \mathrm{w} / \mathrm{v}$, as this solution showed the most linear, i.e. Newtonian, behavior in the viscosity measurement.

$$
\begin{aligned}
& C_{4 m T}=12.0 \times 0.01318=0.316[\mathrm{~Pa}] \\
& C_{8 m T}=24.0 \times 0.01318=0.158[\mathrm{~Pa}]
\end{aligned}
$$




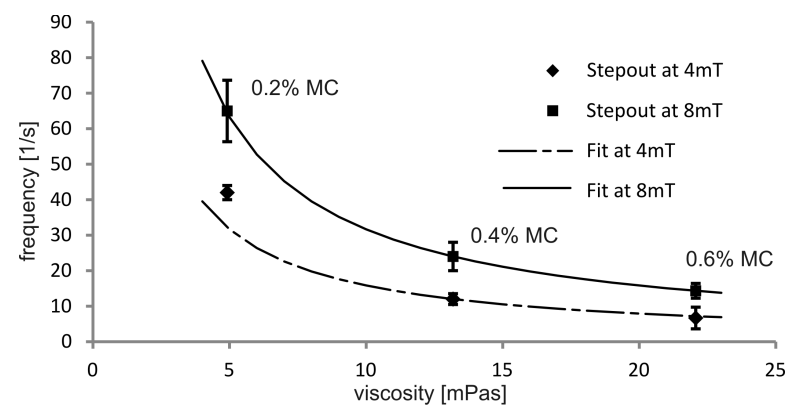

Fig. 8. Stepout frequencies at different viscosities. The values correspond to the averaged values of three measurements and the error bars represent \pm one standard deviation. The line fit is according to (9) with the parameter $C_{4 m T}$ and $C_{8 m T}$ calculated in (10).

It can be seen that the fitted curves $f_{\max \{4 m T, 8 m T\}}=$ $C_{\{4 m T, 8 m T\}} / \eta$ agree well with the experimental data, in particular with the data at $8 \mathrm{mT}$ where the fit is almost perfect. There is, however, some deviation for the data at $4 \mathrm{mT}$ at a low MC concentration. The stepout frequency is a measure of how much torque has to be provided to propel the ABF. As (9) is valid for a Newtonian liquid, it is assumed that the fluid drag that the ABFs encounters in the MC solutions is similar to what is expected in a Newtonian fluid and the Non-Newtonian behavior is not very prominent.

\section{CONCLUSIONS}

ABFs are magnetically actuated microrobots that mimic the propulsion method of bacteria such as the E. coli by using a helical shape for motion generation. Potential applications for these wireless microrobots include medical interventions, for example, as carriers for targeted drug delivery. The challenges lie in actuating ABFs in body fluids, which contain molecules and cells that influence the viscosity and swim behavior of ABFs. Previous publications showed that bacteria can swim more efficiently in structured viscous environments, such as in MC.

In this paper swim tests of $\mathrm{ABF}$ in three concentrations of MC in DI water, namely $0.2,0.4$, and $0.6 \% \mathrm{w} / \mathrm{v}$, have been presented. The viscosities were measured to be approximately 5,13 and $22 \mathrm{mPa} \cdot \mathrm{s}$. Initial results imply that the microrobots swim better in the high concentration MC $(0.4$ and $0.6 \% \mathrm{w} / \mathrm{v})$ than in the lower $0.2 \% \mathrm{w} / \mathrm{v} \mathrm{MC}$ concentration. This result agrees with the behavior reported for bacteria. Furthermore, the result indicates that ABFs can swim well in viscosities over 20 times that of water (approximately $1 \mathrm{mPa} \cdot \mathrm{s}$ at room temperature). This compares favorably with viscosities encountered in some of the fluids in the human body, such as blood (approximately $4 \mathrm{mPa} \cdot \mathrm{s}$ ) and cerebrospinal fluid (approximately $1 \mathrm{mPa} \cdot \mathrm{s}$ ). As the microstructures (molecules, cells) in body fluids vary from the MC structure, further investigations are necessary in order to make a conclusive statement on the swimming capabilities of ABFs in a specific body fluid environment.

\section{ACKNOWLEDGMENT}

The authors would like to thank Phillip Rüst from the Institute of Mechanics at ETH Zurich, Switzerland for his help with the viscosity measurements. We would also like to thank Christos Bergeles for providing the image of the screw-type robot in porcine vitreous.

\section{REFERENCES}

[1] B. J. Nelson, I. K. Kaliakatsos, J. J. Abbott, Microrobots for Minimally Invasive Medicine, Ann. Rev. Biomed. Eng., vol. 12, p. 55-85, 2010

[2] C. Bergeles, M. Kummer, B. E. Kratochvil, C. Framme, B. J. Nelson, Steerable Intravitreal Inserts for Drug Delivery: In Vitro and Ex Vivo Mobility Experiments, Proc. Int. Conf. Medical Image Computing and Computer Assisted Invervention, Toronto, September 2011

[3] S. Pané, O. Ergenemann, K. Silvaraman, T. Lühmann, H. Hall, B. J. Nelson, Strategies for Drug-Delivery and Chemical Sensing using Biomedical Microrobts, Proc. IEEE Int. Conf. Nano/Molecular Medicine and Engineering, Hongkong/Macau, December 2010

[4] A. R. Pries, D. Neuhaus, P. Gaehtgens, Blood Viscosity in the Tube Flow: Dependence on Diameter and Hematocrit, Am. J. Physiol.-Heart Circul. Physiol., vol. 263, no. 6, pp. H1770, 1992

[5] L. G. Bloomfield, I. H. Johnston, L. E. Bilston, Effects of Proteins, Blood Cells and Clucose on the Viscosity of Cerebrospinal Fluid, Pediatr. Neurosurg., vol. 28, no. 5, pp. 246, 1998

[6] M. Kummer, J. J. Abbott, S. Dinser, B. J. Nelson, Artificial Vitreous Humor for In Vitro Experiments, Proc. IEEE Int. Conf. Engineering in Medicine and Biology Society, Lyon, August 2007

[7] L. Zhang, J. J. Abbott, L. X. Dong, B. E. Kratochvil, D. J. Bell, B. J. Nelson, Artificial Bacterial Flagella: Fabrication and Magnetic Control, Appl. Phys. Lett., vol. 94, no. 064107, 2009

[8] L. Zhang, J. J. Abbott, L. X. Dong, K. E. Peyer, B. E. Kratochvil, H. X. Zhang, C. Bergeles, B. J. Nelson, Characterizing the Swimming Properties of Artificial Bacterial Flagella, Nano Lett., vol. 9, no. 10, p. 3663-3667, 2009

[9] L. Zhang, K. E. Peyer, B. J. Nelson, Artificial bacterial flagella for micromanipulation, Lab Chip, vol. 10, no. 17, p. 2203-2215, 2010

[10] K. E. Peyer, L. Zhang, B. J. Nelson, Localized Non-contact Manipulation Using Artificial Bacterial Flagella, Appl. Phys. Lett., vol. 99, no. 174101,2011

[11] E. Purcell, Life at low Reynolds number, Am. J. Phys., vol. 45, no. 1, p. 3-11, 1977

[12] E. Purcell, The Efficiency of Propulsion by a Rotating Flagellum, Proc. Nat. Acad. Sci. USA, vol. 94, no. 21, p. 11307-11311, 1997

[13] H. C. Berg, L. Turner, Movement of microorganisms in viscous environments, Nature, vol. 278, p. 349-351, 1979

[14] Y. Magariyama, S. Kudo, A Mathematical Explanation of an Increase in Bacterial Swimming Speed with Viscosity in Linear-Polymer Solutions, Biophys. J., vo. 83, no. 2, p. 733-739, 2002

[15] A. M. Leshansky, Enhanced low-Reynolds-number propulsion in heterogeneous viscous environments, Phys. Rev. E, vol. 80, no. 5, p. 1-13, 2009

[16] B. Liu, T. R. Powers, K. S. Breuer, Force-free swimming of a model helical flagellum in viscoelastic fluids, Proc. Nat. Acad. Sci., vol. 108, p. 19516-19520, 2011

[17] E. Lauga, Propulsion in a viscoelastic fluid, Phys. Fluids, vol. 19, pp. 083104, 2007

[18] T. Gillespie, The viscosity of concentrated polymer solutions, Polym. Sci., vol. 46 , no. 148 , p. 283-302, 1960

[19] R. P. Chhabra, Non-Newtonian Fluids: An Introduction, in Rheology of Complex Fluids, A. P. Deshpande, P. B. Sunil Kumer, J. M. Krishnan, Springer Science+Business Media, New York, 2010

[20] C. N. LaFratta, J. T. Fourkas, T. Baldacchini, R. A. Farrer, Multiphoton Fabrication, Angew. Chem. Int. Ed., vol. 46, pp. 6238, 2007

[21] S. Tottori, L. Zhang, F. Qiu, K. Krawczyk, A. Franco-Obregón, B. J. Nelson, Magnetic Helical Micromachines: Fabrication, Controlled Swimming, and Cargo Transport, Adv. Mater., vol. 24, no. 6, p. 811816, 2012

[22] K. E. Peyer, L. Zhang, B. E. Kratochvil, B. J. Nelson, Non-ideal Swimming of Artificial Bacterial Flagella Near a Surface, Proc. Int. Conf. Robotics and Automation, Anchorage, May 2010, p. 96-101

[23] E. Lauga, W. R. DiLuzio, G. M. Whitesides, H. A. Stone, Swimming in Circles: Motion of Bacteria near Solid Boundaries, Biophys. J., vol. 90, no. 2, p. 400-412, 2006 